\title{
Randomly Amplified Polymorphic DNA (RAPD) Variation among and within Artichoke (Cynara scolymus L.) Cultivars and Breeding Populations
}

\author{
Jan Tivang, Paul W. Skroch, and James Nienhuis ${ }^{1}$ \\ Department of Horticulture, University of Wisconsin, Madison, WI 53706 \\ Neal De Vos \\ Elkhorn Research and Development Company, P.O. Box 1156, Castroville, CA 95012
}

Additional index words. genetic distance, genetic relationships, multidimensional scaling, sampling variance, genetic diversity, genetic integrity, Cynara cardunculus ssp. flavescens

\begin{abstract}
The magnitude of genetic differences among and heterogeneity within globe artichoke cultivars is unknown. Variation among individual heads (capitula) from three artichoke cultivars and two breeding populations were evaluated using RAPD markers. One vegetatively propagated cultivar ('Green Globe'), two seed-propagated cultivars ('Imperial Star' and 'Big Heart') and two breeding populations were examined. Two to thirteen polymorphic bands were observed for 27 RAPD primers, which resulted in 178 scored bands. Variation was found within and among all cultivars, and breeding populations indicating that all five groups represent heterogeneous populations with respect to RAPD markers. The genetic relationships among individual genotypes were estimated using the ratio of discordant bands to total bands scored. Multidimensional scaling of the relationship matrix showed five independent clusters corresponding to the three cultivars and two breeding populations. The integrity of the five clusters was confirmed using pooled chi-squares for fragment homogeneity. Average gene diversity (Hs) was calculated for each population sample, and a one-way analysis of variance showed significant differences among populations. 'Big Heart' had an Hs value equivalent to the two breeding populations, while clonally propagated 'Green Globe' and seed propagated 'Imperial Star' had the lowest Hs values. The RAPD heterogeneity observed within clonally propagated 'Green Globe' is consistent with phenotypic variability observed for this cultivar. Overall, the results demonstrate the utility of the RAPD technique for evaluating genetic relationships and contrasting levels of genetic diversity among populations of artichoke genotypes.
\end{abstract}

The artichoke (Cynara scolymus) is an outcrossing diploid, $2 \mathrm{n}$ $=2 \mathrm{x}=34$ (Foury, 1967) but its worldwide production is dominated by vegetatively propagated cultivars. Seed-propagated cultivars are rare, as relatively few populations have been developed with commercially acceptable phenotypic uniformity. Knowledge regarding genetic differences among and the magnitude of heterogeneity within cultivars would be useful in plant breeding programs designed to develop more uniform seed-propagated cultivars. Moreover, knowledge of genetic diversity can be used to monitor changes in variability due to prolonged selection and diversity in commercial cultivars where low phenotypic variability is preferred. However, the levels of variation within and among artichoke cultivars is generally unknown. Furthermore, there is almost no knowledge of the overall genetic structure of the artichoke germplasm. Two studies have reported related information. Stevens et al. (1990) evaluated several genera of related thistles, including Cynara, for polyacetylenes. Although their intent was not to study systematic relationships among these genera, the data show considerable variation among genera for these compounds. Hammouda et al. (1993) observed variability among cultivars of artichokes based on HPLC data.

Genetic relationships based on molecular markers have been consistent with expectations based on pedigrees and breeding behavior in many crop species (dos Santos et al., 1994; Nienhuis et al., 1993; Smith et al., 1990). Estimates of genetic variability

Received for publication 5 July 1995. Accepted for publication 13 May 1996. The cost of publishing this paper was defrayed in part by the payment of page charges. Under postal regulations, this paper therefore must be hereby marked advertisement solely to indicate this fact.

${ }^{1}$ To whom reprint requests should be addressed. among genotypes can be obtained using conventional molecular techniques, such as analysis of seed proteins and isozymes; however, results of these analyses often do not reveal sufficient polymorphism to detect genetic variability among genotypes (Goodman and Stuber, 1980; Smith, 1984). The PCR-based RAPD (Williams et al., 1990) molecular markers are relatively easy to generate and inexpensive. Reliability of RAPD bands has been questioned by Smith et al. (1994), Thormann et al. (1994), and Weeden et al. (1992). However, others have found that RAPD data can be reliably generated and scored. Dos Santos et al. (1994) showed that the results of an RFLP analysis of genetic relationships among Brassica oleracea genotypes was consistent with genetic relationships based on RAPD data. Furthermore, the scoring error frequencies were equivalent for the two types of marker data. Skroch and Nienhuis (1995b) and Spooner et al. (1996) showed that the frequency of random errors, regardless of source, was low $(\leq 2 \%)$ among replicate DNA samples scored for RAPD markers.

The objectives of this study were to 1) measure genetic relationships among samples of individuals from five artichoke populations, including three artichoke cultivars, using RAPD molecular markers, 2) determine the genetic integrity of each of the five groups of individual genotypes, and 3) contrast the observed degree of genetic variability within each of the five groups.

\section{Materials and Methods}

Germplasm. Individual heads (capitula) from three commercial cultivars and two breeding populations were used in this study (Table 1). 'Green Globe', which recently accounted for more than $85 \%$ of the artichoke production in the United States (California Artichoke Advisory Board, personal communication), is com- 
Table 1. Artichoke cultivars and populations sampled for analysis with RAPD markers.

\begin{tabular}{lccc}
\hline \hline Entry & Sample size $^{\mathrm{z}}$ & Source & Comments \\
\hline Green Globe & 5 & Elkhorn Research \& Development & Vegetatively propagated cultivar \\
Imperial Star & 7 & University of California & Open-pollinated cultivar \\
Big Heart (XR-1) & 7 & Dole Bakersfield, Inc. & Open-pollinated cultivar \\
5473 & 8 & Elkhorn Research \& Development & Open-pollinated breeding population \\
5931 & 6 & Elkhorn Research \& Development & Open-pollinated breeding population
\end{tabular}

${ }^{\mathrm{z}}$ Number of individual capitula (heads) evaluated

posed of an unknown number of phenotypically similar clones selected within the California industry from germplasm introduced from southern Europe in the nineteenth century. Significant variability for yield and other traits exists among individual clones within 'Green Globe' (De Vos, 1986). The clonally propagated cultivar Green Globe should not be confused with genetically unrelated, seed-propagated cultivars of the same or similar name. The two breeding populations examined in this study, '5473' and '5931', are half-sib families obtained through three and four generations, respectively, of recurrent half-sib selection. Both were derived from an original parent population composed of 100 high-yielding 'Green Globe' clones as described by De Vos (1986). 'Imperial Star' (Schrader and Mayberry, 1992) and 'Big Heart' are seed-propagated cultivars derived from germplasm developed by J.A. Principe, USDA-ARS, Brawley, Calif. Both cultivars are well adapted to southern California; however, their internal quality and gross morphology deviate substantially from 'Green Globe'. 'Green Globe', '5473', and '5931' were sampled by harvesting single capitula from individual plants chosen randomly. Capitula of 'Imperial Star' and 'Big Heart' were obtained from commercial production fields following bulk hydro-cooling and grading. Capitula were arbitrarily picked from four distribution packs for each of these two cultivars.

DNA isolation. Fresh capitulas were stored at 4 to $8{ }^{\circ} \mathrm{C}$ for 1 week and bracts were taken from each sampled capitula, frozen in liquid nitrogen, and then ground with dry ice in a Braun coffee grinder. Ground tissue was combined with CTAB (cetyltrimethylammonium bromide) extraction buffer (Rogers and Bendich, 1988), and DNA was extracted as described in Skroch and Nienhuis (1995a). RAPD reactions were performed using the methods of Skroch and Nienhuis (1995a).
Primer selection. An arbitrary set of four genotypes representing four of the five populations was evaluated for polymorphism using 96 10-base RAPD primers (Operon Technologies, Alameda, Calif.). Twenty-seven primers were selected based on the clarity of polymorphic bands. Operon primers A1, A2, A4, A5, A7, A8, A9, A10, A12, A13, A16, A18, A19, B1, B4, B5, B10, B11, B12, B14, B17, C8, D15, D16, D20, E12, and E14 were used in the analysis.

Genetic relationship. Co-migrating polymorphic fragments ranging from 0.3 to $2.2 \mathrm{~kb}$ were scored for presence (1) or absence (0). This structure of co-migrating fragments with the presentabsent states will henceforth be termed a band. Genetic distance (GD) among the 33 individuals was estimated as the complement to the simple matching coefficient (Gower, 1972). A $33 \times 33$ genetic distance matrix was fitted in two dimensions using the Kruskal monotonic multidimensional scaling (MDS) procedure in Systat 5.2 (Wilkinson, 1992). For a detailed discussion of the MDS procedure see Young (1987).

Band correlation. The magnitude of the simple linear correlation coefficient (Steel and Torrie, 1980) was computed for all pairwise comparisons of polymorphic bands. A reference distribution was generated by randomizing the genotypes within each band, simulating genetic equilibrium. A one-way analysis of variance for distribution of means was performed as well as Levene's and O'Brien's tests of homogeneity of variance (SAS Institute, 1994).

Sampling variance. Sampling variance for GD was measured

Fig. 1. Distribution of the magnitudes of the linear correlations computed for pairwise comparisons of RAPD bands, for all bands (hashed line), unique bands (solid line), and a reference population (shaded) generated by randomizing band scores among genotypes.

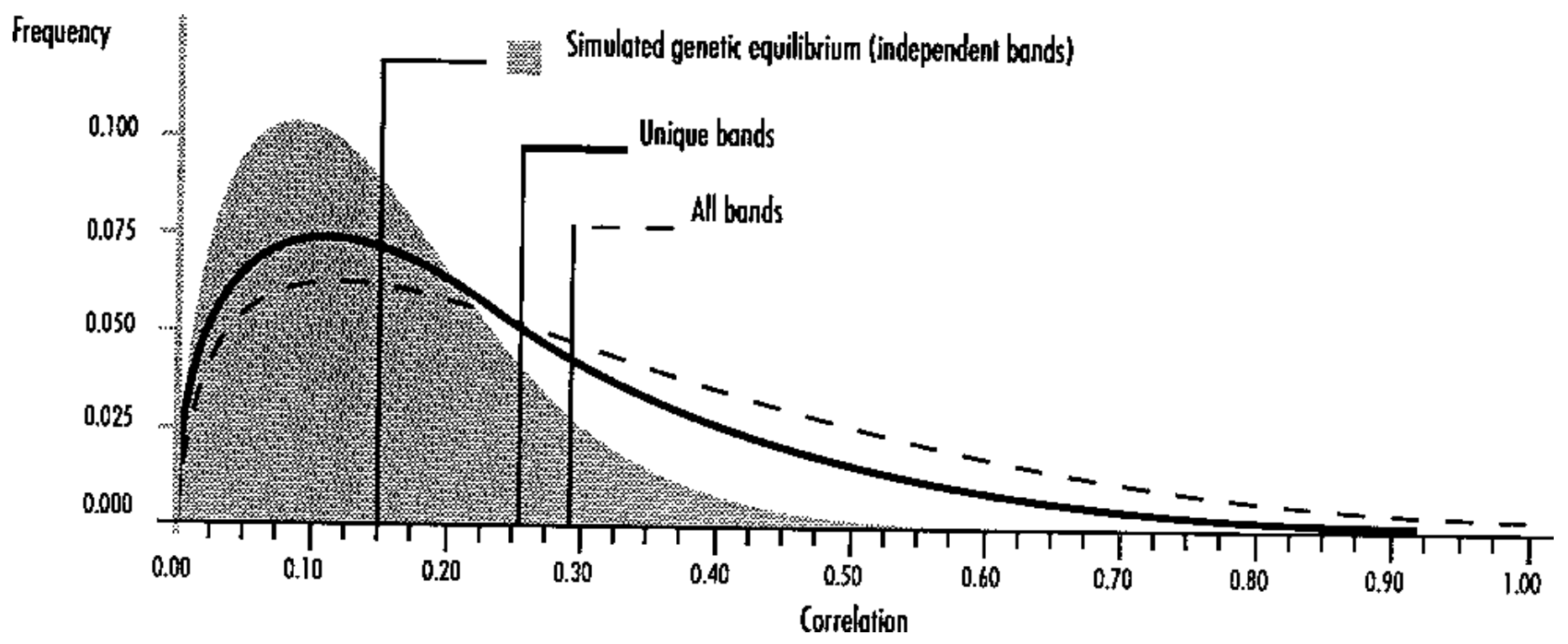




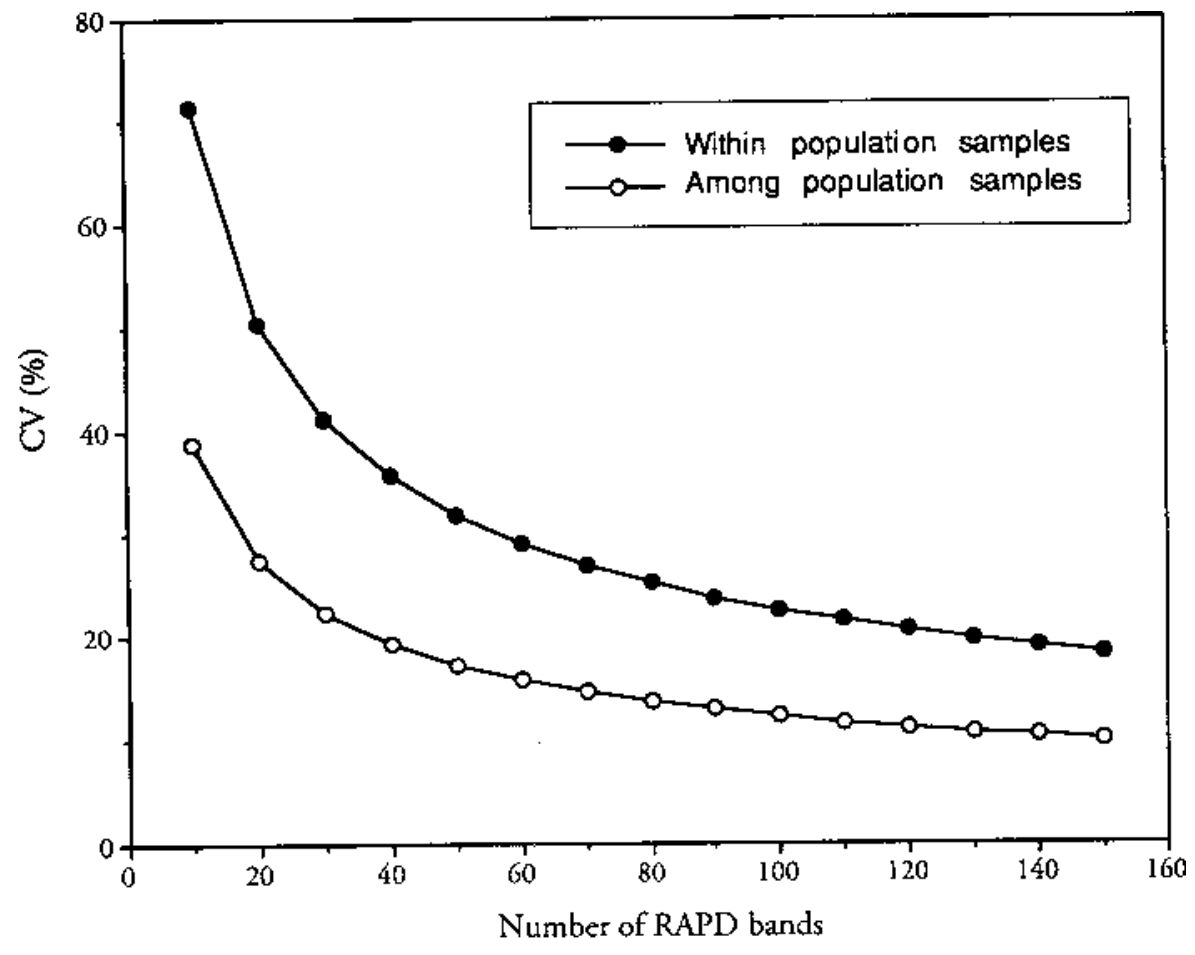

Fig. 2. Relationship between coefficients of variation (cv) and RAPD band sample size for genetic distances between artichoke genotypes computed for pairwise comparison of genotypes within the same population sample and genotypes sampled from different populations. The mean genetic distance computed for pairwise comparison of individuals within populations was 0.16 and between populations was 0.40 .

Genetic diversity. A genetic model of a single locus with two alleles was assumed for each band. Genetic diversity for each population sample was estimated using Nei's gene diversity at a locus, Hs (Nei 1987). For a single locus, gene diversity reduces to $2 \mathrm{pq}$ for a two allele system, where $\mathrm{p}$ and $\mathrm{q}$ are the frequencies of the alternative RAPD amplification states or alleles. Since artichokes are outcrossing and a high degree of heterozygosity was expected, allele frequencies were estimated based on genotypic frequencies. For each RAPD the frequency of the null or homozygous recessive, RAPD genotype, showing absence of amplification, was assumed to be $\mathrm{q}^{2}$, the squared frequency of the null RAPD allele. To reduce bias due to small sample size, $q$ was obtained using Lynch and Milligan's (1994) formula. Computation of $\mathrm{p}$ was obtained by

for within and among population comparisons. For each sample size (10 to 150 bands), 400 random bootstrap samples (sampled with replacement) were independently evaluated. The mean GD was calculated among all possible genotype pairs for each bootstrap sample. The variance among all 400 bootstrap samples for each sample size was evaluated by dividing the standard deviation for each genotype pair with its mean GD, yielding the coefficient of variation (Cv) for that genotype pair. Subsequent summation of all CVs, for specified $\mathrm{n}$, and division by the total number of genotype pairs contrasted, resulted in a mean $\mathrm{CV}$ for within and between population comparisons (Tivang et al., 1994). Alternatively, variances and cvs for genetic distance estimates can be obtained without bootstrapping using the formula for sampling variance given by Skroch and Nienhuis (1995b).

Population integrity. For each pairwise comparison of populations, difference was measured based on individual marker frequency differences. The significance of these observed differences was tested using the chi-square test of goodness of fit (Snedecor and Cochran, 1967) (SAS Institute, 1994). Populations were evaluated pairwise for each band. The mean fragment frequency became, under the null hypothesis, the expected fragment frequency. A band was regarded polymorphic if the mean fragment frequency was not fixed (i.e., 1 or 0 ). Chisquare values were pooled over all polymorphic bands. Degrees of freedom were similarly pooled. Bands with a high degree (>20\%) of missing data were excluded. Degrees of freedom for a paired contrast were lost if the expected value (frequency $\times$ number of individual plants) fell below one. All such observations were grouped into a single class with one degree of freedom (Snedecor and Cochran, 1967).

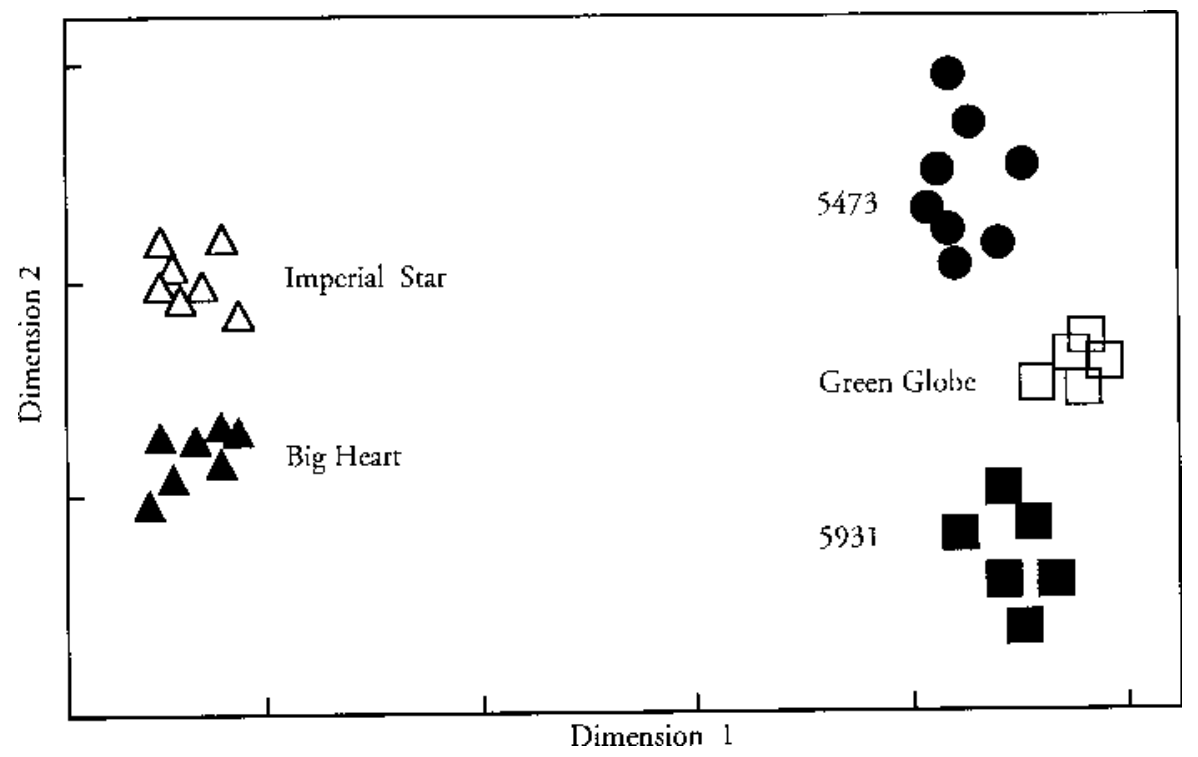

subtraction $(p=1-q)$. A one-way analysis of variance for cultivar differences was performed for Hs, the mean number of polymorphic bands per primer, and Hs using only bands polymorphic within population samples. Fisher's LSD was used to identify specific differences.

Eight, seven, six, seven, and five clones were evaluated for '5473', 'Big Heart', '5931', 'Imperial Star', and 'Green Globe', respectively. Thus, since unequal sample sizes were used, an additional analysis was performed to determine whether unequal sample size had biased the results of the first analysis. The five

Fig. 3. Plot of individual artichoke genotypes by first and second dimension coordinates resulting from a multi-dimensional scaling (MDS) analysis of the genetic distance matrix derived from RAPD marker data. Membership in each of five groups is indicated. 
populations were compared for samples of five genotypes, which was the largest sample size that could be uniformly applied across populations. There are 56 unique subsets of five individual clones from '5473', 21 from 'Big Heart', 6 from '5931', 21 from 'Imperial Star' and only 1 from 'Green Globe'. Thus, there are a total of 56 $\times 21 \times 6 \times 21 \times 1=148,176$ possible unique combinations of samples containing five individuals from each population. ANOVAs were computed (as described above for $\mathrm{Hs}$ ) for a random sample of 2000 of these 148,176 possible unique combinations.

\section{Results and Discussion}

RAPD polymorphisms. Twenty-seven primers were scored for 2 to 13 polymorphic bands per primer, resulting in a total of 178 bands. The best four primers, A10, A12, A18, and B17 accounted for $13,11,10$, and 10 RAPD bands respectively. At least one polymorphic RAPD was found for all 528 pairwise comparisons of genotypes. All but one pair of genotypes differed for at least three bands and all but three pairs of genotypes differed for at least five polymorphic RAPD bands. These results indicate that RAPD marker variation can be easily detected within and among artichoke cultivars and indicates that the three cultivars and the two breeding populations used in this study should be considered genetically heterogeneous populations, with respect to RAPD markers.

Independence. The mean correlation among all bands was 0.29 (Fig. 1, dashed line). In the absence of mapped markers, 28 perfectly correlated band pairs were assumed to represent the same region of the genome. One member of each perfectly correlated pair was excluded from all subsequent analyses, resulting in 150 unique bands with a mean correlation of 0.26 (Fig. 1, solid line). The assumption that perfectly correlated markers (over 33 genotypes divided into 5 independent populations) represent linked loci seems justified considering the outcrossing nature of artichokes and the low likelihood of two unlinked markers having a perfectly correlated distribution in a segregating population. An excess of perfectly correlated RAPD band pairs was similarly reported by Skroch and Nienhuis (1995a), and random sets of RAPD markers have been found to cluster on the bean genetic map (P. Skroch, personal communication). The observed independence was compared to a reference population simulating genetic equilibrium, which had a mean correlation of 0.15 (Fig. 1, gray area). A one-way analysis of variance indicated significant differences among the mean correlations. A pairwise $t$ test between the mean correlation for all and unique bands was significant. Tests of homogeneity of variance also indicated highly significant differences among the three distributions. The distribution shows that removal of correlated bands increases the level of independence. However, as would be expected for a population that is not in random mating equilibrium, greater correlation among the bands was still present relative to the reference population.

Sampling variances. Sampling variance associated with genetic relationships occurs when discrepancies between a random subset of molecular marker loci and all available loci are detected (Tivang et al., 1994). Relationships among populations evaluated with 150 bands yielded a CV of about $10 \%$ (Fig. 2). For genetic distances computed for within population comparisons, the corresponding mean CV was $18.45 \%$, based on all 150 RAPD bands. This reduced level of precision can be attributed to fewer polymorphic bands available and the reduced level of polymorphism within any one population. The results suggest that to establish genetic relationships within populations, additional primers would have to be evaluated. An estimate of 510 polymorphic bands would be required for a $10 \% \mathrm{CV}$ based on the logarithmic transformation of the values plotted in Fig. 2 (Tivang et al., 1994). However, even for small populations, this would be an impractical number of markers to evaluate. An alternative strategy for improving the precision of genetic distance estimates for relatively closely related genotypes would be to preselect sets of markers polymorphic within the targeted subpopulation. Fewer markers would then have to be evaluated since distances among genotypes would be greater, similar to distances among population samples in this study, and $\mathrm{CV}$ s would more rapidly decrease with band sample size, as shown in Fig. 2.

Genetic relationships. The relationships among sampled capitula were displayed based on an MDS analysis of the $33 \times 33$ matrix of genetic distances (Fig. 3.). The proportion of variance explained by the first two MDS dimensions $\left(R^{2}\right)$ was $97.8 \%$ with a stress factor of 0.097 , which indicates a relatively good fit between the two dimensional representation of relationships among genotypes and the original distance matrix. Inspection of the MDS plot showed five discrete clusters corresponding to the five populations. The first dimension indicated two major groups: one including 'Green Globe', '5473', and '5931' and the other including 'Imperial Star' and 'Big Heart'. The second dimension separated these two major groups into five discrete clusters. This result is consistent with the known pedigree of the breeding populations, '5473' and '5931', with their parents consisting of high-yielding clones from 'Green Globe'. Moreover, 'Green Globe' is known to be morphologically distinct from 'Imperial Star' and 'Big Heart'.

Table 2. Pairwise comparison of samples from five artichoke populations based on mean genetic distance between population samples and chi-square analysis of fragment frequencies.

\begin{tabular}{|c|c|c|c|c|}
\hline \multirow[b]{2}{*}{ Comparison } & \multirow[b]{2}{*}{$\mathrm{GD}^{\mathrm{y}}$} & \multicolumn{3}{|c|}{ Fragment frequency comparison ${ }^{z}$} \\
\hline & & $\chi^{2}$ & $\mathrm{df}$ & $P>\chi^{2}$ \\
\hline Imperial Star vs. Big Heart & 0.28 & 181 & 109 & 0.000 \\
\hline Imperial Star vs. Green Globe & 0.54 & 340 & 112 & 0.000 \\
\hline Imperial Star vs. 5473 & 0.42 & 324 & 122 & 0.000 \\
\hline Imperial Star vs. 5931 & 0.50 & 383 & 127 & 0.000 \\
\hline Big Heart vs. Green Globe & 0.48 & 292 & 116 & 0.000 \\
\hline Big Heart vs. 5473 & 0.43 & 295 & 132 & 0.000 \\
\hline Big Heart vs. 5931 & 0.46 & 306 & 129 & 0.000 \\
\hline Green Globe vs. 5473 & 0.31 & 132 & 102 & 0.024 \\
\hline Green Globe vs. 5931 & 0.28 & 134 & 105 & 0.031 \\
\hline 5473 vs. 5931 & 0.34 & 178 & 123 & 0.001 \\
\hline
\end{tabular}

${ }^{\mathrm{Z}}$ Pooled chi-square test for each pairwise comparison under the null hypothesis of no differences in RAPD fragment frequencies between groups. ${ }^{\mathrm{y}}$ Mean of genetic distance estimates for pairwise comparison of genotypes from different groups. 
Population integrity. Germplasm identification is important for protection and monitoring of proprietary cultivars or breeding populations. Note that none of the sampled individuals in this study had identical RAPD profiles. Thus, a unique RAPD fingerprint exists for each individual sampled in this study. However, variation among individual genotypes also makes it more difficult to define unique RAPD profiles for the groups of individuals that make up cultivars or populations. To evaluate the uniqueness of population samples, fragment frequencies were compared, on a band-by-band basis, using pooled chi-squares (Table 2). These frequencies in essence define the population. All 10 pairwise comparisons of samples from the five populations were contrasted and all were significantly different. This result verifies the observed relationships revealed by the MDS plot, where 'Green Globe' is intermediate between ' 5473 ' and '5931' and all five populations form distinct clusters.

Alternatively, mean genetic distances could have been used to estimate differences among population samples (Nienhuis et al., 1993). Mean genetic distances were computed (Table 2). However, using genetic distance means is similar to using bulked tissue or tissue from a single plant (Nienhuis et al., 1993; Smith et al., 1990; Spooner et al., 1996; Thormann et al., 1994) to determine population identity because it masks population heterogeneity. Analysis of fragment frequencies is a more direct method of evaluating population integrity, which does not mask intrapopulation variability. The impact of selection on populations can be interpreted directly in terms of changes in marker frequencies. Breeding and deliberate selection result in gene frequency changes and corresponding marker frequency changes occur due to drift or linkage to selected genes. Selection and drift can explain the distinctiveness of ' 5473 ', '5931', and 'Green Globe', despite the fact that '5473' and '5931' are half-sib families derived from a set of selected high yielding 'Green Globe' clones.

Genetic diversity. Genetic diversity is important to the breeder since the genetic variation present in breeding populations ultimately determines the potential for making gains from selection. Mean genetic distance within populations (Table 3 ) showed that the population samples surveyed in this study have a varying degree of variation and could be ranked. A oneway analysis of variance using $\mathrm{Hs}$ values (Table 3 ) showed a significant $F$ ratio (13.55) indicating the presence of significantly different levels of diversity among populations. Fisher's LSD showed no significant difference between any nearest ranked neighbor except for '5473' and 'Big Heart' $(P>0.046)$.

Similar results were obtained when the diversity analysis was based on genetic distance, gene diversity, and the number of polymorphic bands per primer. However, when comparisons of diversity were restricted to bands polymorphic within populations, significant differences among populations were not found $(\mathrm{F}=$ 1.14, Table 3). Furthermore, eight individuals were sampled from the ' 5473 ' population, which was the most diverse, while only five individuals were sampled from 'Green Globe', which ranked the lowest in terms of RAPD marker diversity. Thus, average gene diversity was correlated with the number of bands polymorphic within a cultivar and the number of individuals in a population. These relationships are important because, by chance, more polymorphic bands and, hence, a greater estimated gene diversity might be expected for larger samples of individuals, especially for small sample sizes. Taken together, these results suggest that differences among populations may be an artifact of sample size. To test this hypothesis, an additional analysis was performed in which samples of equal size were taken from each population. ANOVAs were computed for Hs for a random sample of 2000 sets containing five individuals from each population. All F tests were highly significant. Furthermore, in $97.6 \%$ (1952) of the analyses, the populations were ranked as in Table 3 . Remarkably, the mean F, averaged over all 2000 ANOVAs, was identical to the F value previously obtained based on unequal sample sizes. Thus, we can conclude that differences among these population samples were not effected by unequal sample size.

However, in this experiment, only five to eight individuals were evaluated per population. The low sample sizes, especially for 'Green Globe', may have narrowed the range of detectable diversity in a sample. Such samples could only detect the most polymorphic loci with reasonable probability. Thus, due to small sample size, the ranking of the five populations, based on the samples studied, must be considered preliminary. In addition, since one consequence of the heterogeneous and heterozygous nature of artichoke cultivars may be the divergence of different populations of the same cultivar, surveys of larger numbers of populations are recommended. For example, 'Green Globe' populations with similar cultivation histories may be genetically distinct from other 'Green Globe' populations. The results presented here demonstrate that RAPD markers can be used to address such questions.

We would expect uniform commercial populations to show reduced variability for markers. 'Imperial Star' and 'Green Globe' show similar low levels of RAPD variability. The variability observed in the vegetatively propagated 'Green Globe' was attributed to heterogeneity among clones, which is consistent with its multiclonal composition (De Vos, 1986). However, '5473' and

Table 3. Analysis of within population variation measured by mean genetic distance, average gene diversity, and number of polymorphic bands per primer based on RAPD marker data collected for samples of individuals from five artichoke populations. A one-way analysis of variance was performed for all measurements except genetic distance.

\begin{tabular}{|c|c|c|c|c|c|c|c|c|}
\hline & \multicolumn{5}{|c|}{ Population } & \multicolumn{3}{|c|}{ ANOVA } \\
\hline & 5473 & Big Heart & 5931 & Imperial Star & $\overline{\text { Green Globe }}$ & $\mathrm{df}$ & F ratio & $P>\mathrm{F}$ \\
\hline$\overline{\text { Within population } \mathrm{GD}^{\mathrm{z}}}$ & 0.240 & 0.185 & 0.145 & 0.093 & 0.084 & & & \\
\hline $\mathrm{Hs}^{\mathrm{y}}$ & $0.271 \mathrm{a}^{\mathrm{x}}$ & $0.215 b$ & $0.170 \mathrm{bc}$ & $0.117 \mathrm{~cd}$ & $0.093 \mathrm{~d}$ & 4 & 13.55 & 0.0000 \\
\hline Hs for polymorphic loci ${ }^{w}$ & 0.473 & 0.474 & 0.481 & 0.486 & 0.539 & 4 & 1.14 & 0.3379 \\
\hline Number of bands ${ }^{\mathrm{v}}$ & 86 & 68 & 53 & 36 & 25 & & & \\
\hline Bands per primer $^{\mathrm{u}}$ & $3.190 \mathrm{a}$ & $2.520 \mathrm{ab}$ & $1.960 \mathrm{bc}$ & $1.33 \mathrm{~cd}$ & $0.93 \mathrm{~d}$ & 4 & 7.72 & 0.0000 \\
\hline
\end{tabular}

${ }^{\mathrm{z}}$ Mean genetic distance between individuals within a population sample.

yNei's gene diversity averaged over 150 RAPD loci, five loci were excluded for 'Green Globe' due to missing data.

${ }^{\mathrm{x}}$ Means are significantly different by $\mathrm{LSD}_{0.05}$ when seperated by different letters.

wNei's gene diversity computed for each population sample based on the subset of bands polymorphic within that group.

vNumber of RAPD bands polymorphic within each population sample.

uThe mean number of polymorphic bands scored per primer for 27 primers evaluated. 
'5931' were selected from a base population consisting of 100 high-yielding 'Green Globe' clones, yet the variability indicated for these two breeding populations was greater than that for 'Green Globe'. Furthermore, some RAPD alleles present in the breeding populations were not present in the sample of five 'Green Globe' clones. There are two possible explanations for this result. First, the five green globe clones used in this study were not selected out of the same population of 100 'Green Globe' clones used to develop the two breeding populations. Second, a sample of only five individuals likely resulted in a failure to sample the full range of diversity represented by the 100 clones from which the two breeding populations were developed.

The marginal difference $(P>0.046)$ between '5473' and 'Big Heart' for Nei's Hs and the absence of a difference between 'Big Heart' and '5931' shows the level of variability available in 'Big Heart' as a population. This variability is consistent with broad adaptation of the 'Big Heart' population. The high degree of variability in the breeding population ' 5473 ', and to a lesser extent ' 5931 ', is consistent with additional refinements in progress before their release. Additional selection with in these breeding populations would also improve their RAPD integrity and better differentiate them from 'Green Globe'.

\section{Conclusions}

RAPD data were used to estimate genetic relationships within and among samples of individuals representing five artichoke populations. The pooled chi-square analysis of RAPD marker frequencies and the MDS analysis of the genetic distance matrix showed all population samples to be clearly defined. Increasing the number of polymorphic primers would improve estimates of genetic relationships among individual capitula within a population. Based on small samples of individuals, significant differences among populations for Nei's Hs were found. However, increasing the number of individuals per population would facilitate more precise estimates of population diversity. All individuals sampled from all populations were distinguishable with the RAPD markers used in this study. The level of variability present in artichoke cultivars has significant implications for cultivar identification and maintenance of cultivar integrity. The results demonstrate the utility of RAPD markers for contrasting levels of diversity within artichoke populations.

\section{Literature Cited}

De Vos, N.E. 1986. Field improvement through single-plant selection in globe artichoke (Cynara scolymus L.) 'Green Globe'. HortScience 21:662. (Abstr.)

dos Santos, J.B., J. Nienhuis, P. Skroch, J. Tivang, and M. Slocum. 1994. Comparison of RAPD and RFLP genetic markers in determining genetic similarity among Brassica oleracea L. genotypes. Theor. Appl. Genet. 87:909-915.

Foury, C. 1967. Study of the floral biology of the artichoke Cynara scolymus L. with application to selection. Part I (in French). Ann. Amelior. Plantes 17:357-373.

Goodman, M.M. and C.W. Stuber. 1980. Genetic identification of lines and crosses using isoenzymes electrophoresis. Proc. 35th Annu. Corn Sorghum Res. Conf. 35:10-31.

Gower, J.C. 1972. Measurement of taxonomic distance and their analysis, p. 1-24. In: J.S. Weiner and J. Huizinga (eds.). The assessment of population affinities in man. Clarendon Press, Oxford.

Hammouda, R.M., M.M. Seif El-Nasr, S.I. Ismail, and A.A. Shahat. 1993. Quantitative determination of the active constituents in Egyptian cultivated Cynara scolymus. Intl. J. Pharmacog. 31:299-304.

Lynch, M and G. Milligan. 1994. Analysis of population genetic structure with RAPD markers. Mol. Ecol. 3:91-99.

Nei, M. 1987. Molecular evolutionary genetics. Columbia Univ. Press, New York. p. 180-189.

Nienhuis, J., M.K. Slocum, D.A. De Vos, and R. Muren. 1993. Genetic similarity among Brassica oleracea L. genotypes as measured by restriction fragment length polymorphisms. J. Amer. Soc. Hort. Sci. 118:298-303.

Rogers, S.O. and A.J. Bendich. 1988. Extraction of DNA from milligram amounts of fresh, herbarium and mummified plant tissue. Plant Mol. Biol. 5:69-76.

SAS Institute. JMP/Statistics made visual. version 3.1. SAS Inst., Cary, N.C.

Schrader, W.L. and K.S. Mayberry. 1992. 'Imperial Star' artichoke. HortScience 27:375-376.

Skroch, P. and J. Nienhuis. 1995a. Qualitative and quantitative characterization of RAPD variation among snap bean (Phaseolus vulgaris L.) genotypes. Theor. Appl. Genet. 91:1078-1085.

Skroch, P. and J. Nienhuis. 1995b. Impact of scoring error and reproducibility of RAPD data on RAPD based estimates of genetic distance. Theor. Appl. Genet. 91:1086-1091.

Smith, J.J., J.S. Scott-Craig, J.R. Leadbetter, G.L. Bush, D.L. Roberts, and D.W. Fulbright. 1994. Characterization of random amplified polymorphic DNA (RAPD) products from Xanthomonas campestris and some comments on the use of RAPD products in phylogenetic analysis. Mol. Phylo. Evol. 3:135-145.

Smith, J.S.C. 1984. Genetic variability within US hybrid maize: Multivariate analysis of isozyme data. Crop Sci. 24:1041-1046.

Smith, O.S., J.S.C. Smith, S.L. Bowen, R.A. Tenborg, and S.J. Wall. 1990. Similarities among a group of elite maize inbreds as measured by pedigree, $\mathrm{F}_{1}$ grain yield, grain yield heterosis and RFLPs. Theor. Appl. Genet. 80:833-840.

Snedecor, G.W. and W.G. Cochran. 1967. Statistical methods. Iowa Univ. Press, Ames.

Spooner, D.M., J.G. Tivang, J. Nienhuis, J.P. Miller, D.S. Douches, and A.M. Contreras. 1996. Comparison of four molecular markers in evaluating accessions of the wild potato relatives Solanum sectionEtuberosum (subgenus Potatoe). Theor. Appl. Genet. (In press.)

Steel R.G.D. .and J.H. Torrie. 1980. Principles and procedures of statistics. McGraw-Hill, New York. p. 272.

Stevens, K.L., S.C. Witt, and C.E. Turner. 1990. Polyacetylenes in related thistles of the subtribes centaureinae and carduinae. Biochem. Syst. Ecol. 18:229-232.

Tivang, J.G., J. Nienhuis, and O.S. Smith. 1994. Sampling variance of molecular marker data sets using the bootstrap. Theor. Appl. Genet 89:259-564.

Thormann, C.E., M.E. Ferreira, L.E.A. Camargo, J.G. Tivang, and T.C. Osborne. 1994. Comparison of RFLP and RAPD genetic relationships within and among cruciferous species. Theor. Appl. Genet. 88:973-980.

Weeden, N.F., G.M. Timmerman, M. Hemmat, B.E. Kneen, and M.A. Lodhi. 1992. Inheritance and reliability of RAPD markers, p 12-17. In: Applications of RAPD technology to plant breeding. Crop Sci. Soc. Amer., Madison Wis.

Wilkinson, L. 1992. SYSTAT: Statistics. ver. 5.2. SYSTAT, Evanston Ill. Williams, J.G.K., A.R. Kubelik, K.J. Livak, J.A. Rafalski, and S.V. Tingey. 1990. DNA polymorphisms amplified by arbitrary primers are useful as genetic markers. Nucleic Acids Res. 18:6531-6535.

Young, F.W. 1987. Part I: History, p. 15-42; Part II, Theory, p. 43-158. In: R.M. Hamer (ed.). Multidimensional scaling: History, theory, and applications. Lawrence Erlbaum Associates, Hillsdale, N.J. 